\title{
A novel reversible thermo-swelling hydrogel
}

\author{
J. Q. Wang ${ }^{1,2 *}$, M. Satoh ${ }^{2}$ \\ ${ }^{1}$ School of Materials Science and Engineering, Beijing Institute of Technology, Beijing, 100081, P. R. China \\ ${ }^{2}$ Department of Chemistry and Materials Science, Tokyo Institute of Technology, Tokyo, 152-8550, Japan
}

Received 23 March 2010; accepted in revised form 7 May 2010

\begin{abstract}
A novel reversible thermo-swelling gel was prepared from poly(vinyl alcohol)-trimellitate (PVA-T) by crosslinking with ethylene glycol diglycidyl ether (EGDGE). Only in the presence of sulfate anion, this polymer gel showed a significant and reversible swelling behavior with increasing the temperature from 5 to $40^{\circ} \mathrm{C}$, and vice versa, probably due to the scission and formation of the inter- and/or intramolecular hydrogen-bondings (HBs) between the carboxyls on the side groups. The involvement of inter- and/or intramolecular HBs for the thermo-swelling behavior was also suggested by a significant dependence on $\mathrm{HCl}$ concentration of the swelling degree. In addition, the swelling reversibility and reproducibility were confirmed via the temperature jump between 5 and $40^{\circ} \mathrm{C}$, well satisfying for a candidate as a thermosensitive material.
\end{abstract}

Keywords: polymer gels, reversible thermo-swelling, Hofmeister Series

\section{Introduction}

In a hydrogel, a few interactions, such as van der Waals, ionic, hydrophobic and hydrogen-bonding, are exerted among macromolecules, water and other substances to determine the swelling degree. Any change in the interactions will cause a variation in the gel size, sometimes dramatically as the volume phase transition [1]. Thus, an external stimulus such as temperature, $\mathrm{pH}$, ionic strength, solvent, and so on may change the gel size via alteration in the interactions.

Among the external stimuli, the temperature effect has been most extensively studied and hydrogels prepared from poly(N-isopropylacrylamide) (PNIPAAm) must be the most typical as a thermoresponsive polymer gel. This polymer has a lower critical solution temperature (LCST) in water, and the LCST could be changed to different extents by various modifications [2]. In addition, it has also been found that poly(N,N-diethylacrylamide), poly(N-vinylcaprolactam), poly(2-isopropyl-2-oxa- zoline) and poly(vinyl methyl ether) have LCST in water [3-6]. For these thermo-responsive polymers, one common feature is that the hydrophobic interaction drives the aggregation of macromolecules to induce the collapse above the LCST, and another is that the swollen (or hydrated) state can be recovered by decreasing the temperature, namely, the swelling-collapse cycle can be reversibly repeated. The hydrogen bond is also sensitive to temperature and may be utilized as a main interaction causing a thermo-swelling of relevant hydrogels. In fact, Tanaka et al. [1] firstly found that poly(acrylamide)/ poly(acrylic acid) interpenetrating network (PAAm/ PAA IPN) gel revealed a reversible discontinuous transition with temperature. Further they also proved that hydrogen-bonding (HB) is the driving force for the transition on the basis of the urea effect because $\mathrm{HB}$ is the only attractive force in PAAm/PAA IPN to induce the collapse at low temperature. To our best knowledge, this somewhat sophisticatedly prepared hydrogel is the only one

*Corresponding author, e-mail: jqwang @ bit.edu.cn

(C) BME-PT 
example that shows a reversible gel swelling and deswelling with temperature increasing and decreasing, respectively, on the basis of HB interaction.

Recently, Suzuki and coworkers reported that poly(sodium acrylate) (PSA) and poly(N-isopropylacrylamide-co-sodium acrylate) (NIPA-SA) gels deswelled under repeated exchange of external water, which was attributed to protonation of the carboxyl groups and the resultant formation of inter- and/or intramolecular HBs between-COOHs. Further interestingly, the gels showed a discontinuous reswelling at a certain elevated temperature because of the destruction of HBs [7, 8]. However, the thermo-swelling was not reversible; namely, with decreasing the temperature the gel kept the swollen state.

In the present letter, we report a novel reversible thermo-swelling gel prepared from poly(vinyl alcohol)-trimellitate (PVA-T) by a chemical crosslinking. This polymer gel is collapsed in water due to intermolecular $\mathrm{HBs}$ among - $\mathrm{COOHs}$ at the side groups $[9,10]$, and shows no appreciable thermoswelling. In the presence of sulfate anion, however, a significant and reversible swelling occurs with increasing the temperature, and vice versa. Although this swelling behavior is not a volume phase transition, the PVA-T gel is a first example showing a reversible thermo-swelling as a hydrogel that was prepared with a simple chemical crosslinking.

\section{Experimentals}

\subsection{Preparation of PVA-T hydrogel samples}

According to a procedure that was reported previously [10], PVA-T polymer was prepared by the esterification of PVA (degree of hydrolysis: 99-100 mol\%, $M_{w}: 86000$, Acros) and trimellitic anhydride (Tokyo Chemical Industry) in dimethyl sulfoxide (Kanto Chemical), under the catalysis of 4-(N,N-dimethylamino)pyridine (Kanto Chemical) and triethylamine (Kanto Chemical). In this work, we used a PVA-T polymer with a rather high esterification degree (ED), i.e. $82 \mathrm{~mol} \%$, to get hydrogels crosslinked with ethylene glycol diglycidyl ether (Aldrich). The detailed procedure is given elsewhere [10], with the exception that the crosslinker was used as $10 \mathrm{~mol} \%$ with respect to the amount of PVA-T side groups in this work. The rod-type gel samples were prepared in glass capillaries $(\varphi=0.69 \mathrm{~mm})$ at $30^{\circ} \mathrm{C}$ for $24 \mathrm{~h}$. After taken out, they were equilibrated to pure water and then to $1 \mathrm{mM} \mathrm{HCl}$ solution for several days with daily exchanging of the external solution. With this pretreatment, the carboxyl groups introduced to PVA at the side group (ca. $1.6 \mathrm{~mol}$ per vinyl alcohol residue) were protonized and kept in the undissociated state.

\subsection{Temperature dependence of hydrogel swelling}

The equilibrium gel diameter in $1 \mathrm{mM}$ aqueous $\mathrm{HCl}$ was first determined as $d_{0}$ with an optical microscope (DIAPHOT 200, NIKON) at room temperature $\left(\mathrm{ca} .25^{\circ} \mathrm{C}\right)$. Then the samples were immersed in different salt solutions (0.1-1 $\mathrm{M} \mathrm{Li}_{2} \mathrm{SO}_{4}, 1 \mathrm{M}$ $\mathrm{LiCl}$ or $\mathrm{LiSCN}$ ) containing $1 \mathrm{mM} \mathrm{HCl}$ or in pure water. In those experiments, the gel samples were small enough compared to the immersing solution (gel diameter: ca. $0.4 \mathrm{~mm}$, length: ca. $3 \mathrm{~mm}$, solution volume: $4 \mathrm{ml}$ ), and held in screw cap vials with $10 \mathrm{ml}$ capacity. The swelling degree, defined as $d / d_{0}(d$ : gel diameter in the acidic salt solutions or in pure water), was measured as a function of temperature; the respective gel samples were first equilibrated in a water bath at $40 \pm 0.5^{\circ} \mathrm{C}$ for $48 \mathrm{~h}$, and then the temperature was decreased by $5^{\circ} \mathrm{C}$ down to $5 \pm 0.5^{\circ} \mathrm{C}$, by changing the bath temperature. It is worth noting that the gels were kept at a certain temperature for $24 \mathrm{~h}$ and the swelling was equilibrated. As for the sulfate system, the temperature dependence of the swelling degree was measured as a function of the $\mathrm{Li}_{2} \mathrm{SO}_{4}$ concentration (0.1-1 $\mathrm{M}$ with $1 \mathrm{mM} \mathrm{HCl})$ and $\mathrm{HCl}$ concentration (0.1-5 mM with $0.5 \mathrm{M} \mathrm{Li}_{2} \mathrm{SO}_{4}$ ). Aside from the swelling measurement with the gradual temperature change, response and reversibility of the swelling degree to a temperature jump were measured by setting a gel sample, which had been measured in $0.5 \mathrm{M} \mathrm{Li}_{2} \mathrm{SO}_{4} / 1 \mathrm{mM} \mathrm{HCl}$ at $5 \pm 0.5^{\circ} \mathrm{C}$, in a water bath of $40 \pm 0.5^{\circ} \mathrm{C}$. After $24 \mathrm{~h}$, the gel sample was set in another water bath of $5 \pm 0.5^{\circ} \mathrm{C}$. This temperature jump was repeated twice as $\left(5^{\circ} \mathrm{C}\right)-40^{\circ} \mathrm{C}-$ $5^{\circ} \mathrm{C}-40^{\circ} \mathrm{C}-5^{\circ} \mathrm{C}$.

\section{Results and discussion}

Hydrogel swelling properties reflect the nature of crosslinkages including chemical and physical ones within gels. In the present study, the latter involves 
HBs between $-\mathrm{COOH}$ groups, $-\mathrm{OH}$ groups, $-\mathrm{COOH} /-\mathrm{OH},-\mathrm{COOH}(-\mathrm{OH}) / \pi$ electrons and $\pi-\pi$ stacking of the benzene rings. All of them, especially the first one between - $\mathrm{COOH}$ groups, seemed to be responsible for the collapse of PVA-T gels in pure water [10] and such a low water content as $25 \mathrm{wt} \%$. Further, the observed marked swelling in sulfate solutions was ascribed to the breakage of the inter- and/or intramolecular HB between the carboxyl groups [10]. On the other hand, the chemical crosslinkage with 10 mol\% EGDGE may only restrict the swelling degree of the PVA-T gel in a non-specific way. Therefore we focus on the discussion of HBs relevant to - $\mathrm{COOHs}$ instead of the chemical crosslinkage in the present study.

As reported in our previous papers $[9,10]$, the PVA-T gels swelled significantly only in sulfate solutions and we called this unique behavior antiHofmeister Series property. In addition to the anion-specificity, an appreciable cation-specificity was also observed. Thus, $\mathrm{Li}_{2} \mathrm{SO}_{4}$ revealed more significant salting-in effect than $\mathrm{Na}_{2} \mathrm{SO}_{4}$, because

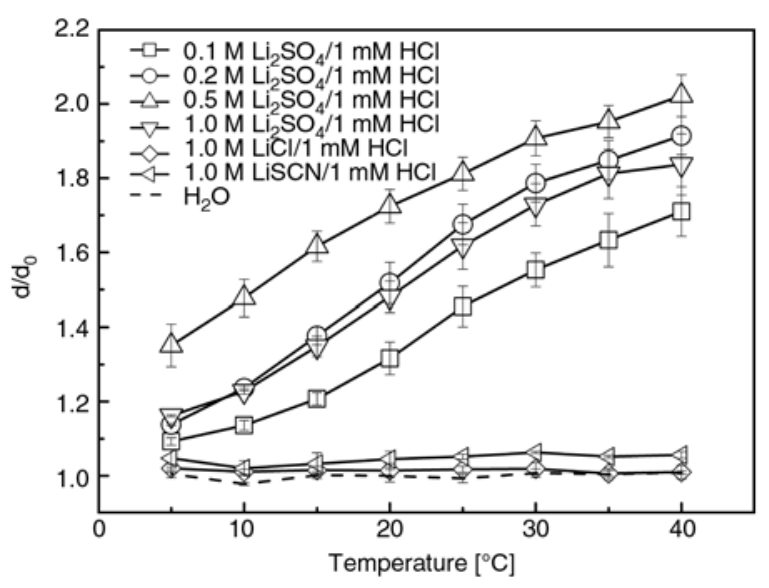

Figure 1. The swelling degree of PVA-T hydrogels in different aqueous solutions as a function of temperature of the much stronger interaction of $\mathrm{Li}^{+}$with the substituted trimellitic acid groups than that of $\mathrm{Na}^{+}$ [10]. Therefore lithium salts/PVA-T gel systems were chosen as the most typical one to examine the thermo-sensitivity of the relevant gel. Swelling degrees of PVA-T hydrogels in various lithium salt solutions containing $1 \mathrm{mM} \mathrm{HCl}$ and in pure water were investigated as a function of temperature, as shown in Figure 1. The hydrogels significantly swelled with temperature only in the presence of $\mathrm{Li}_{2} \mathrm{SO}_{4}$, while in pure water and $1 \mathrm{M} \mathrm{LiCl}$ or LiSCN solution, no appreciable changes were observed with temperature changes.

The significant swelling of PVA-T gels only in sulfate systems might be ascribed to the specific stabilization of hydrogen-bonding hydration $(\mathrm{HBH})$ to the acidic protons of the carboxyl groups by the sulfate anion, as shown in Figure 2 as a schematic for the salt- and thermo-swelling mechanism $[9,10]$. The stabilization of the $\mathrm{HBH}$ to the $-\mathrm{COOH}$ protons should be favorable for breaking those HBs involving carboxyls to cause the salt-induced gel swelling. Thus, the present 'thermo-swelling' that was observed only in the presence of sulfate anion may be safely ascribed to the scission of the intraand/or intermolecular HBs. In fact, no other reasonable explanation seems to be given for the sulfate anion effect to induce the thermo-swelling. The insensitivity to temperature change in the absence of the sulfate anion, in turn, strongly suggests that the inter- and/or intramolecular HBs are too strong to be broken without the help of $\mathrm{SO}_{4}^{2-}$. Namely, for the HBs to be effectively broken by heating, the presence of sulfate anion seems to be prerequisite. It is known that the parent polymer PVA is largely subject to salting-out effect induced by $\mathrm{SO}_{4}^{2-}$ [11]. Thus, the great salting-in effect of this anion
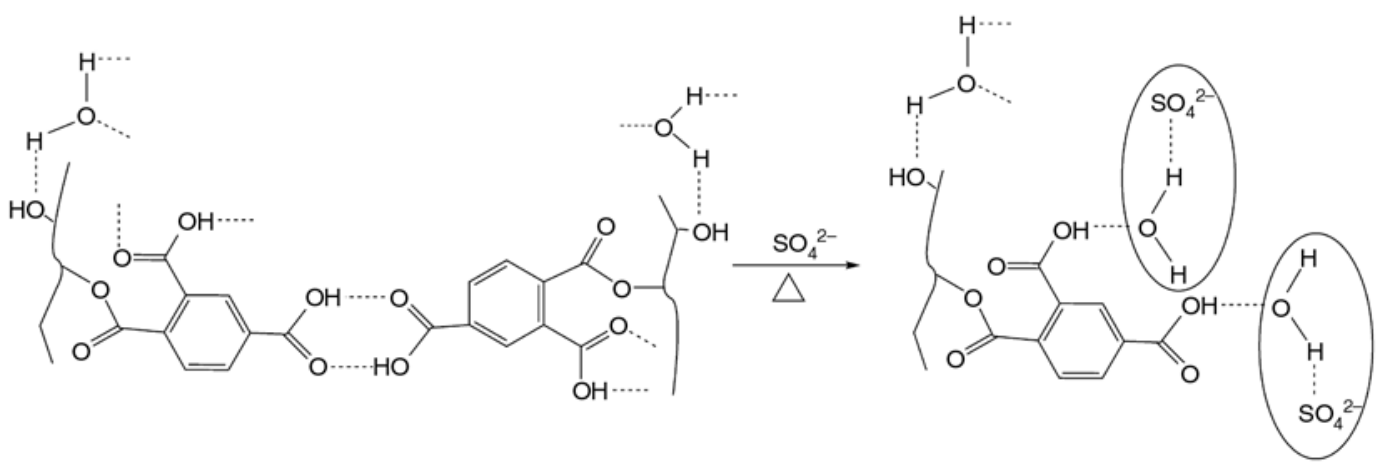

Figure 2. The scission of HBs involving - $\mathrm{COOH}$ groups in the presence of $\mathrm{SO}_{4}^{2-}$ during heating. Here the $\mathrm{HBHs}$ to the acidic protons are significantly stabilized by $\mathrm{SO}_{4}^{2-}$ because the partial negative charges on the water oxygen is enhanced [12]. 
observed for the PVA-T hydrogel must be ascribed to the trimellitic residue introduced to PVA. The salting-in effect may be attributed to the stabilization of $\mathrm{HBH}$ to carboxylic acid proton by $\mathrm{SO}_{4}^{2-}$ via enhancement of electron pair donicity (EPD) of the hydration water, because the partial negative charge on the water oxygen is enhanced via ionic hydration to small anions [12]. As a matter of fact, the improvement of PVA-T hydration in sulfate systems and the temperature effect are also proved by FTIR and NMR, which will be published in our forthcoming paper.

As for the effect of $\mathrm{SO}_{4}^{2-}$ concentration, the maximum swelling emerged at $0.5 \mathrm{M}$ at any temperature. The deswelling at $1.0 \mathrm{M}$ relative to $0.5 \mathrm{M}$ results from the salting-out effect to the unesterified PVA OH group and the vinyl main chain [10]. Of course, the deswelling may be considered as a result of enhanced osmotic pressure from inside out because the salting-out of the sulfate means that the salt ions are rejected from around the polymer to reduce the concentration in gel phase compared with that in the external solution. Comparing the swelling degrees variation of PVA-T hydrogels with temperature, the ratio at 40 to $5^{\circ} \mathrm{C}$ are $2.8,2.5$, 3.0 and 2.7 for samples swollen in $0.1,0.2,0.5$ and $1.0 \mathrm{M} \mathrm{Li}_{2} \mathrm{SO}_{4}$ solution, respectively. Thus, the thermo-swelling profiles are nearly parallel and slightly sigmoidal irrespective of the sulfate concentrations studied.

In order to examine whether the breakage/formation of $\mathrm{HBs}$ between - $\mathrm{COOH}$ groups is the driving force for the thermo-responsibility of PVA-T gel in $\mathrm{Li}_{2} \mathrm{SO}_{4}$ solutions, dissociation of the $-\mathrm{COOH}$ groups was changed by altering $\mathrm{HCl}$ concentration in $0.5 \mathrm{M} \mathrm{Li}_{2} \mathrm{SO}_{4}$ solution. As shown in Figure 3, the thermo-swelling behavior was only observed in the presence of moderate concentrations of $\mathrm{HCl}$. It is noteworthy that the swelling degrees of samples in 0.1 and $0.5 \mathrm{mM} \mathrm{HCl}$ systems become saturated at higher temperatures (nearly equal values are observed at over $30^{\circ} \mathrm{C}$ ). This may be ascribed to almost a complete breakage of inter- and intramolecular HBs in the hydrogels due to the stabilization of the $\mathrm{HBH}$ by sulfate anion as well as to inherent partial dissociation of the carboxyl groups. (At $\mathrm{pH}=4-3.3$, the dissociation degree of $-\mathrm{COOH}$ would be less than $50 \%$.) On the other hand, at $5 \mathrm{mM} \mathrm{HCl}$, no appreciable swelling was observed. Under such a rather acidic condition $(\mathrm{pH} \sim 2.3)$, the

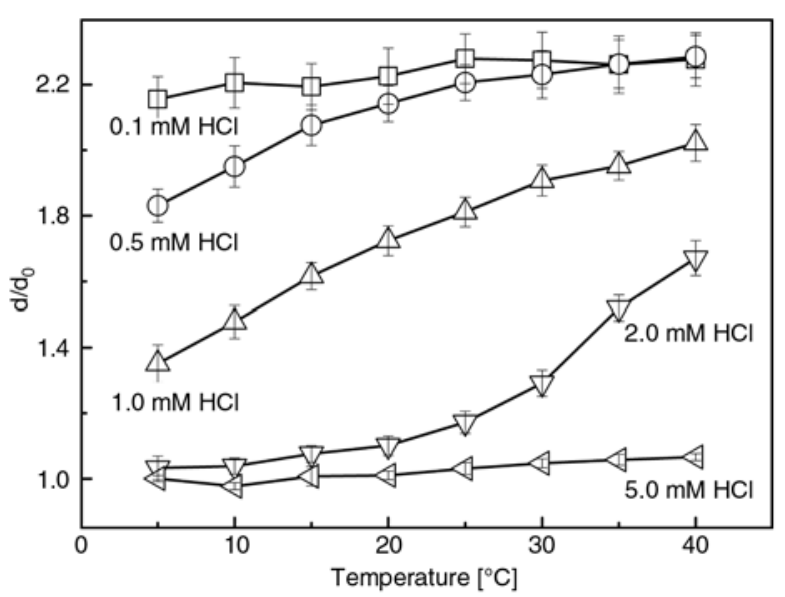

Figure 3. The influence of $\mathrm{HCl}$ concentration on the temperature-dependence of PVA-T hydrogels swollen in $0.5 \mathrm{M} \mathrm{Li}_{2} \mathrm{SO}_{4}$ system

dissociation may be almost suppressed to stabilize the intermolecular HBs. Thus, these behaviors suggest that the thermo-swelling found for the PVA-T hydrogels occurs with a subtle balance of two counteracting factors: concentrations of $\mathrm{HCl}$ $\left(\mathrm{H}_{3} \mathrm{O}^{+}\right)$and $\mathrm{Li}_{2} \mathrm{SO}_{4}\left(\mathrm{SO}_{4}^{2-}\right)$. An increment of the former would suppress the dissociation of $-\mathrm{COOH}$ groups and favor the inter- and/or intramolecular HBs, while the latter may cause the scission of the HBs via stabilization of the HBH to the acidic proton. As a result of the subtle balance between the two factors, the present gel may also be taken as a $\mathrm{pH}$-sensitive material; in fact, e.g. at $30^{\circ} \mathrm{C}$, the water content increases from ca. $25 \%$ in $5 \mathrm{mM} \mathrm{HCl}$ to ca. $90 \%$ in $0.5 \mathrm{mM} \mathrm{HCl}$.

Finally, taken the hydrogel swollen in $0.5 \mathrm{M}$ $\mathrm{Li}_{2} \mathrm{SO}_{4} / 1 \mathrm{mM} \mathrm{HCl}$ system as an example, the reversibility of the thermo-swelling was tested by repeating a temperature jump between 5 and $40^{\circ} \mathrm{C}$ (Figure 4). When the equilibrated gel samples at $5^{\circ} \mathrm{C}$ were placed into the bath of $40^{\circ} \mathrm{C}$, they swelled quickly to the value of $d / d_{0}=2.10$ after $3.5 \mathrm{~h}$, approaching to the equilibrium value of 2.14 after $24 \mathrm{~h}$. Then the highly swollen sample at $40^{\circ} \mathrm{C}$ was put into the environment of $5^{\circ} \mathrm{C}$, the significant deswelling was observed within $6 \mathrm{~h}$. The first and the second swelling degrees obtained after $24 \mathrm{~h}$ kept nearly constant at 40 and $5^{\circ} \mathrm{C}$, respectively. Although the kinetics of the swelling-deswelling processes is rather slow, the reversibility and the reproducibility are well satisfying for a thermo-sensitive gel material that significantly swells upon heating. 


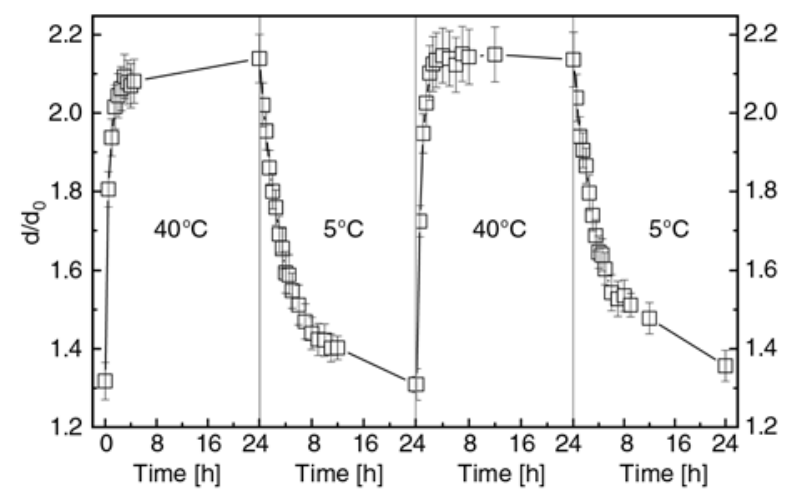

Figure 4. The swelling sensitivity to temperature jump between 5 and $40^{\circ} \mathrm{C}$ for PVA-T hydrogel swollen in $0.5 \mathrm{M} \mathrm{Li}_{2} \mathrm{SO}_{4} / 1 \mathrm{mM} \mathrm{HCl}$ solution

\section{Conclusions}

In the temperature range from 5 to $40^{\circ} \mathrm{C}$, PVA-T hydrogels showed reversible and continuous thermoswelling behavior only in the presence of $\mathrm{SO}_{4}^{2-}$. This rather specific behavior was ascribed to scission and formation of the inter- and/or intramolecular HBs in the course of the heating and the cooling, respectively. Although the PVA-T hydrogel only continuously responded to temperature under the $\mathrm{Li}_{2} \mathrm{SO}_{4} / \mathrm{HCl}$ conditions tested in the present study, it may be expected that a reversible volume-phase transition driven by HB may be realized through optimizing the composition of swelling medium such as sulfate salt and $\mathrm{HCl}$. To delve into the mechanism of salt- and thermo-swelling of PVA-T gels in sulfate solutions, a spectroscopic study with NMR and FTIR are now being undertaken, which will be published in our coming paper.

\section{Acknowledgements}

This study was supported by Grant-in-Aid for foreign postdoctoral fellows (19.07798) from Japan Society for the Promotion of Science (JSPS).

\section{References}

[1] Ilmain F., Tanaka T., Kokufuta E.: Volume transition in a gel driven by hydrogen bonding. Nature, 349, 400-401 (1991). DOI: $10.1038 / 349400 \mathrm{a} 0$

[2] Dimitrov I., Trzebicka B., Müller A. H. E., Dworak A., Tsvetanov C. B.: Thermosensitive water-soluble copolymers with doubly responsive reversibly interacting entities. Progress in Polymer Science, 32, 1275-1343 (2007).

DOI: 10.1016/j.progpolymsci.2007.07.001
[3] Idziak I., Avoce D., Lessard D., Gravel D., Zhu X. X.: Thermosensitivity of aqueous solutions of $\operatorname{poly}(\mathrm{N}, \mathrm{N}$ diethylacrylamide). Macromolecules, 32, 1260-1263 (1999).

DOI: $\underline{10.1021 / \mathrm{ma981171f}}$

[4] Laukkanen A., Valtola L., Winnik F. M., Tenhu H.: Formation of colloidally stable phase separated poly(Nvinylcaprolactam) in water: A study by dynamic light scattering, microcalorimetry, and pressure perturbation calorimetry. Macromolecules, 37, 2268-2274 (2004).

DOI: $\underline{10.1021 / \mathrm{ma0351241}}$

[5] Diab C., Akiyama Y., Kataoka K., Winnik F. M.: Microcalorimetric study of the temperature-induced phase separation in aqueous solutions of poly(2-isopropyl-2-oxazolines). Macromolecules, 37, 2556$2562(2004)$

DOI: $\underline{10.1021 / \mathrm{ma} 0358733}$

[6] Meeussen F., Bauwens Y., Moerkerke R., Nies E., Berghmans H.: Molecular complex formation in the system poly(vinyl methyl ether)/water. Polymer, 41, 3737-3743 (2000).

DOI: 10.1016/S0032-3861(99)00513-3

[7] Hirashima Y., Sato H., Suzuki A.: ATR-FTIR spectroscopic study on hydrogen bonding of poly $(N$-isopropylacrylamide-co-sodium acrylate) gel. Macromolecules, 38, 9280-9286 (2005).

DOI: $\underline{10.1021 / \mathrm{ma} 051081 \mathrm{~s}}$

[8] Sato H., Hirashima Y., Suzuki A.: Reswelling transition of poly(sodium acrylate) gels due to destruction of hydrogen bonds observed by ATR FTIR spectroscopy. Journal of Applied Polymer Science, 105, 3811-3816 (2007).

DOI: 10.1002/app.26626

[9] Mori M., Wang J., Satoh M.: Anti-Hofmeister series properties found for a polymer having a $\pi$ electron system and acidic protons. Colloid and Polymer Science, 287, 123-127 (2009).

DOI: $\underline{10.1007 / \mathrm{s} 00396-008-1965-0}$

[10] Wang J., Satoh M.: Novel PVA-based polymers showing an anti-Hofmeister series property. Polymer, 50, 3680-3685 (2009).

DOI: 10.1016/j.polymer.2009.05.050

[11] Muta H., Miwa M., Satoh M.: Ion-specific swelling of hydrophilic polymer gels. Polymer, 42, 6313-6316 (2001). DOI: $10.1016 / \mathrm{S} 0032-3861(01) 00098-2$

[12] Muta H., Kawauchi S., Satoh M.: Ion effects on hydrogen-bonding hydration of polymer an approach by 'induced force model'. Journal of Molecular Structure: THEOCHEM, 620, 65-76 (2003). DOI: $10.1016 / \mathrm{S} 0166-1280(02) 00585-7$ 